\title{
IMPACTO ECONÓMICO DE LA PREMATUREZY LAS MALFORMACIONES CONGÉNITAS SOBRE EL COSTO DE LA ATENCIÓN NEONATAL
}

\author{
Hugo Salinas $P^{1,2}$, Jaime Albornoz $V^{1}{ }^{1}$, Marcia Erazo $B .{ }^{2 a}$, Jorge Catalán M. ${ }^{1}$, María \\ Eugenia Hübner G. ${ }^{1}$, Jessica Preisler $R .{ }^{1}$, M. Eugenia Juárez $H{ }^{1}$, Rodolfo Ide $V .{ }^{1}$, Sergio \\ Carmona $G^{3}$, Manuela Fernández $B .^{b}$ \\ ${ }^{1}$ Departamento de Obstetricia y Ginecología, Hospital Clínico de la Universidad de Chile. ${ }^{2}$ Escuela de Salud Pública de \\ la Universidad de Chile. ${ }^{3}$ Departamento de Obstetricia y Ginecología, Hospital Clínico San Borja Arriarán.
}

a Enfermera Universitaria, b Ingeniero Comercial, Hospital Clínico de la Universidad de Chile.

\section{RESUMEN}

Objetivos: Calcular los costos de la atención neonatal de recién nacidos prematuros y en portadores de malformaciones congénitas mayores compatibles con la vida. Pacientes y Método: Estudio retrospectivo efectuado en el Departamento de Ginecología y Obstetricia del Hospital Clínico de la Universidad de Chile, en 82 recién nacidos menores de 34 semanas de gestación y en 14 con malformaciones congénitas mayores, de más de 37 semanas de gestación, compatibles con la vida, atendidos entre enero y diciembre de 2004. Resultados evaluados son los costos de la atención neonatal subdivididos en componentes. Resultados: El costo promedio de la atención neonatal en recién nacidos menores de 34 semanas fue igual a $\$ 2.519 .508$, en menores de 32 semanas igual a $\$ 3.766 .999$, en menores de 1500 gramos igual a $\$ 12.017 .650$ y en portadores de malformaciones congénitas mayores compatibles con la vida de $\$ 30.967 .180$. El día cama representa el componente más significativo dentro cada paquete con más del $60 \%$ del costo promedio. Conclusiones: El costo de la atención neonatal de prematuros menores de 34 semanas o portadores de malformaciones congénitas mayores compatibles con la vida es mayor al contemplado en los paquetes de prestaciones a todo evento, representando el día cama su componente más significativo.

\section{PALABRAS CLAVES: Atención neonatal, costo de salud, prematurez, malformaciones congénitas mayores}

\section{SUMMARY}

Objective: To calculate the cost involved in the neonatal care of premature or live born babies carriers of mayor congenital abnormalities compatible with life. Patients and method: Retrospective study who analyzed 82 premature live born of less than 34 weeks and 14 live born carriers of mayor congenital abnormalities compatible with life, from January to December 2004, at the Maternity Ward from the University of Chile Clinical Hospital. The outcome measures were the neonatal care average cost package subdivided by components. The cost was expressed in chilean currency. Results: Neonatal care average cost was $\$ 2.519 .508$ in live born of less than 34 weeks, $\$ 3.766 .999$ in less than 32 weeks, $\$ 12.017 .650$ in less than 1500 grams babies and $\$ 30.967 .180$ in carriers of mayor congenital abnormalities. In bed day cost represents the most significant component from the package, representing more than $60 \%$ of its total cost. 
Conclusions: Neonatal care cost of premature live born of less than 34 weeks or carriers of mayor congenital abnormalities compatible with life is higher than the maximum cost considered in the every-event health packages, representing in bed day its most significant component.

\section{KEY WORDS: Neonatal care, health cost, premature, mayor congenital abnormalities}

\section{INTRODUCCIÓN}

El pago asociado a diagnóstico (PAD) es una estrategia destinada a cancelar a los establecimientos de salud por la actividad que realizan en reemplazo del tradicional pago de los gastos en personal, bienes y servicios de consumo e inversión que se hace a dichos centros independiente de las acciones de salud que realizan.

El modelo es simple y consiste básicamente en definir una prestación, estandarizar sus componentes, fijar los costos por ítem y finalmente el costo total de la prestación. Se considera una distribución normal, asumiéndose que en el 95\% de las veces los costos involucrados en la prestación otorgada están dentro del valor asignado, $2,5 \%$ bajo la misma y $2,5 \%$ sobre ésta.

El gestor clínico organiza la prestación en base a recomendaciones que surgen de la evidencia médica, toma las medidas necesarias para disminuir la variabilidad en la práctica clínica y audita las eventuales dispersiones del paquete de prestaciones.

En obstetricia, todas las prestaciones que se otorgan pueden ser razonablemente estandarizadas, en el marco de una mezcla de riesgos que no implique una tendencia a actuar con casos tan complejos, que el $2,5 \%$ de las veces en que el paquete debiera costar más que lo asumido previamente sobrepase largamente dicho porcentaje.

La estrategia PAD será asumida en Chile, en el marco del Acceso Universal con Garantía Explícitas (AUGE), con un valor determinado por el comprador, a ser negociado con prestadores públicos y privados que será previamente establecido y con una mezcla calculada de riesgos. ¿Qué pasará entonces en aquellos establecimientos con una mezcla de riesgos superior al estándar? Una alternativa es subsidiar a aquellos centros de atención de alta complejidad cuya mezcla de riesgos se aparte de lo definido para el país. Otra opción es pagar por servicios cuando el valor del paquete se aleje, auditoría mediante, de la lógica de la construcción del mismo. Finalmente, se pudieran construir paquetes de prestaciones, con distintos componentes y por ende con diferentes costos según el cuadro en cuestión. Dos situaciones complican la estrategia de paquetes de prestaciones en el campo obstétrico: la prematurez y las malformaciones congénitas mayores compatibles con la vida.

El objetivo del presente estudio es calcular los costos de la atención de recién nacidos prematuros o con malformaciones congénitas mayores compatibles con la vida y que representan para cualquier servicio clínico del país situaciones económicas muy difícil de asumir bajo la lógica de la construcción de paquetes de prestaciones a todo evento.

\section{MATERIAL Y MÉTODOS}

Se elaboró un paquete tipo que considera sólo prestaciones y costos de atención de recién nacidos prematuros menores de 34 semanas de gestación y recién nacidos mayores de 37 semanas con malformaciones congénitas compatibles con la vida.

Los componentes del paquete que fueron considerados incluyeron: consultas médicas, días camas, exámenes de laboratorio, exámenes de imagen, intervenciones quirúrgicas, procedimientos médicos y de enfermería, medicamentos, insumos y prestaciones especiales no otorgadas en nuestro establecimiento.

Este esquema se aplicó a la totalidad de los recién nacidos que fueron atendidos el año 2004 en el Hospital Clínico de la Universidad de Chile: 82 recién nacidos menores de 34 semanas de gestación y 14 recién nacidos con malformaciones congénitas mayores de más de 37 semanas de gestación y compatibles con la vida.

\section{RESULTADOS}

En la Tabla I se aprecia el total de las medias de los costos de las prestaciones otorgadas a los niños prematuros menores de 34 semanas, como asimismo el costo por cada rubro. En ésta se aprecia que el costo medio de la prestación alcanza a $\$ 2.519 .508$, destacando el valor medio del día cama que alcanza a $64,85 \%$ del costo de la prestación media. 
Tabla I

COSTO PROMEDIO DE PRESTACIÓN A PREMATUROS MENORES DE 34 SEMANAS EN 2004

\begin{tabular}{lrr}
\hline Componentes & Costo $(\$)^{*}$ & $\%$ \\
\hline Consultas & 35.939 & 1,43 \\
Días cama & 1.634 .003 & 64,85 \\
Exámenes de laboratorio & 57.429 & 2,28 \\
Imagenología & 55.844 & 2,22 \\
Intervenciones quirúrgicas & 253.434 & 10,06 \\
Procedimientos & 43.353 & 1,72 \\
Medicamentos & 233.039 & 9,25 \\
Insumos & 206.467 & 8,19 \\
\hline Total general & 2.519 .508 & 100 \\
\hline
\end{tabular}

${ }^{*} \$=$ pesos chilenos $(1$ US $\$=559,83$ pesos chilenos al $30 \mathrm{de}$ diciembre de 2004).

En la Tabla II se aprecia cómo estos costos aumentan, llegando a \$3.766.999 como costo medio total del paquete. Nuevamente el costo de los días camas ocupados representa el mayor costo de la prestación media, elevándose al $68,46 \%$ de la misma. Se aprecia mayor costo para cada uno de los componentes del paquete cuando el análisis es realizado en prematuros menores de 32 semanas de gestación al momento del nacimiento en comparación con aquellos que tienen menos de 34 semanas.

En la Tabla III se aprecian los costos cuando la variable de estudio considera a los niños con peso inferior a los 1.500 gramos al momento del nacimiento y que sobrevivieron hasta el alta médica del establecimiento. En éstos, el costo total medio

Tabla II

COSTO PROMEDIO DE PRESTACIÓN A PREMATUROS MENORES DE 32 SEMANAS EN 2004

\begin{tabular}{lrr}
\hline Componentes & Costo $(\$)^{*}$ & \multicolumn{1}{c}{$\%$} \\
\hline Consultas & 53.128 & 1,41 \\
Días cama & 2.578 .734 & 68,46 \\
Exámenes de laboratorio & 82.647 & 2,19 \\
Imagenología & 103.832 & 2,76 \\
Intervenciones quirúrgicas & 280.012 & 7,43 \\
Procedimientos & 53.862 & 1,43 \\
Medicamentos & 365.679 & 9,71 \\
Insumos & 249.105 & 6,61 \\
\hline Total general & 3.766 .999 & 100 \\
\hline
\end{tabular}

${ }^{*} \$=$ pesos chilenos $(1$ US $\$=559,83$ pesos chilenos al 30 de diciembre de 2004).
Tabla III

COSTO PROMEDIO DE PRESTACIÓN A PREMATUROS MENORES DE 1500 GRAMOS EN 2004

\begin{tabular}{lrr}
\hline Componentes & Costo $(\$)^{*}$ & \multicolumn{1}{l}{$\%$} \\
\hline Consultas & 133.635 & 1,11 \\
Días cama & 7.720 .260 & 64,24 \\
Exámenes de laboratorio & 245.332 & 2,04 \\
Imagenología & 215.483 & 1,79 \\
Intervenciones quirúrgicas & 333.043 & 2,77 \\
Procedimientos & 411.027 & 3,42 \\
Medicamentos & 1.523 .810 & 12,68 \\
Insumos & 1.038 .584 & 8,64 \\
Prestaciones especiales & 396.476 & 3,30 \\
\hline Total general & 12.017 .650 & 100 \\
\hline
\end{tabular}

${ }^{*} \$=$ pesos chilenos $(1 \mathrm{US} \$=559,83$ pesos chilenos al $30 \mathrm{de}$ diciembre de 2004).

de las prestaciones alcanzan ahora a $\$ 12.017 .650$, representando el costo de los días camas el $64,24 \%$ del costo total de las prestaciones.

La Tabla IV muestra el costo máximo de las prestaciones otorgadas a un niño prematuro menor de 1.500 gramos en el Hospital (\$43.932.072). Un análisis similar fue realizado en nuestro Servicio con los niños malformados, apreciándose el costo medio de las prestaciones para el año 2004, las que alcanzaron a $\$ 30.967 .180$ (Tabla V), representando el costo de día cama el 30,11\% y las prestaciones especiales el $39,62 \%$. El costo máximo de las prestaciones otorgadas a uno de los niños con malformaciones alcanzó a $\$ 160.637 .380$.

Tabla IV

COSTO DE PRESTACIÓN MÁXIMA A PREMATUROS MENORES DE 1500 GRAMOS EN 2004

\begin{tabular}{lrr}
\hline Componentes & Costo $(\$)^{*}$ & $\%$ \\
\hline Consultas & 183.448 & 0,42 \\
Días cama & 31.010 .568 & 70,59 \\
Exámenes de laboratorio & 787.240 & 1,79 \\
Imagenología & 628.316 & 1,43 \\
Intervenciones quirúrgicas & - & - \\
Procedimientos & 1.274 .208 & 2,90 \\
Medicamentos & 5.221 .432 & 11,89 \\
Insumos & 4.034 .860 & 9,18 \\
Prestaciones especiales & 792.000 & 1,80 \\
\hline Total general & 43.932 .072 & 100 \\
\hline
\end{tabular}

* $\$=$ pesos chilenos $(1 \mathrm{US} \$=559,83$ pesos chilenos al $30 \mathrm{de}$ diciembre de 2004). 
Tabla V

\section{COSTO PROMEDIO DE PRESTACIÓN A RECIÉN NACIDOS CON MALFORMACIONES CONGÉNITAS EN 2004}

\begin{tabular}{lrr}
\hline Componentes & Costo $(\$)^{*}$ & \multicolumn{1}{l}{$\%$} \\
\hline Consultas & 366.452 & 1,18 \\
Días cama & 9.324 .405 & 30,11 \\
Exámenes de laboratorio & 495.375 & 1,60 \\
Imagenología & 641.473 & 2,07 \\
Intervenciones quirúrgicas & 1.870 .932 & 6,04 \\
Procedimientos & 1.250 .137 & 4,04 \\
Medicamentos & 2.467 .237 & 7,97 \\
Insumos & 2.282 .966 & 7,37 \\
Prestaciones especiales & 12.268 .203 & 39,62 \\
\hline Total general & 30.967 .180 & 100 \\
\hline
\end{tabular}

* $\$=$ pesos chilenos (1US $\$=559,83$ pesos chilenos al 30 de diciembre de 2004).

\section{DISCUSIÓN}

La Organización Mundial de la Salud (OMS) con el apoyo de centros europeos, ha definido prematurez como aquel recién nacido que nace antes de las 37 semanas cumplidas (1). Sin embargo, la Academia Americana de Pediatría (AAP) propone como definición aquellos recién nacidos (RN) menores de 38 semanas, debido al mayor riesgo de morbilidad existente entre las 37 y 38 semanas.

No obstante, estas definiciones de prematurez parecen insuficientes, porque comprende un grupo muy heterogéneo de niños, cuya edad gestacional, peso de nacimiento, estado nutricional, riesgos y morbi-mortalidad son diferentes, por lo que es necesario catalogarlos en subgrupos. Así surgen los recién nacidos de muy bajo peso de nacimiento (RNMBPN) que corresponden a los menores de 1.500 gramos los recién nacidos de extremo bajo peso de nacimiento (RNEBPN) o prematuros extremos que se refiere a los menores de 1.000 gramos o menores de 28 semanas y el RN micronato o neonato fetal que son aquellos que tienen un peso entre 500 y 750 gramos y constituyen el grupo de mayor riesgo y de mayor costo.

Los RN menores de $1.500 \mathrm{~g}$ constituyen según diferentes publicaciones internacionales alrededor del $1 \%$ de los nacidos vivos. Según un estudio realizado por el Ministerio de Salud de Chile en el año 2000, los menores de $1500 \mathrm{~g}$ alcanzan al $0,92 \%$. Por otra parte, los menores de $1.000 \mathrm{~g}$ constituyen el $0,34 \%$ de los recién nacidos vivos, observándose claras diferencias regio-
Tabla VI

COSTO DE PRESTACIÓN MÁXIMA POR MALFORMACIONES CONGÉNITAS EN 2004

\begin{tabular}{lrr}
\hline Componentes & \multicolumn{1}{c}{ Costo $(\$)^{*}$} & \multicolumn{1}{c}{$\%$} \\
\hline Consultas & 2.961 .060 & 1,84 \\
Días cama & 92.003 .030 & 57,27 \\
Exámenes de laboratorio & 4.521 .995 & 2,82 \\
Imagenología & 4.795 .850 & 2,99 \\
Intervenciones quirúrgicas & 3.686 .120 & 2,29 \\
Procedimientos & 8.848 .660 & 5,51 \\
Medicamentos & 18.311 .310 & 11,40 \\
Insumos & 24.068 .925 & 14,98 \\
Prestaciones especiales & 1.440 .430 & 0,90 \\
\hline Total general & 160.637 .380 & 100 \\
\hline
\end{tabular}

${ }^{*} \$=$ pesos chilenos $(1 \mathrm{US} \$=559,83$ pesos chilenos al $30 \mathrm{de}$ diciembre de 2004).

nales (2). Durante los últimos años, en el Hospital Clínico de la Universidad de Chile ha aumentado el porcentaje de atención de recién nacidos vivos menores de $1500 \mathrm{~g}$, alcanzando durante el año 2004 los menores de $1500 \mathrm{~g}$ y los menores de $1000 \mathrm{~g}$ un $2,9 \%$, y $1,4 \%$, respectivamente.

Lo anterior ha determinado un impacto significativo tanto desde el punto de vista clínico como económico, representando el grupo de recién nacidos prematuros de muy bajo peso aproximadamente el 50 a $70 \%$ de la mortalidad neonatal y el 25 a $30 \%$ de la mortalidad infantil $(3,4,5)$.

En Chile, la sobrevida para los RN menores de $1500 \mathrm{~g}$ es de $69 \%$, con una amplia variabilidad según regiones (44-82\%) (2).

El grupo NEOCOSUR (Neonatología del Cono Sur) integrado por Chile, Perú, Uruguay, Argentina y Brasil, reporta un $73 \%$ de sobrevida, con una variabilidad de 47 a $88 \%$ entre los países que lo integran (6).

En el Hospital Clínico de la Universidad de Chile, entre el año 2000 y 2004, la sobrevida de RN menores de $1500 \mathrm{~g}$ fue de $72,4 \%$, destacando que, entre los recién nacidos de este grupo de peso, se atendió un porcentaje alto de niños menores de $1000 \mathrm{~g}$ que concentraron la mayor mortalidad. La sobrevida alcanzada en el grupo de RN entre $1000 \mathrm{~g}$ y $1500 \mathrm{~g}$ resultó mayor al $90 \%$ (datos no publicados).

Sin embargo, la edad gestacional y el peso de nacimiento de los RN prematuros no sólo son importantes como factores predictivos de sobrevida neonatal, sino que también están relacionados en forma inversa con la duración de la hospitaliza- 
ción. Es así como el promedio de días de hospitalización para un RN entre las 26 a 27 semanas es de 100 días (85 a 156 días), a las 28 semanas es de 93 días (58 a 166 días), entre las 29 y 30 semanas de 50 días, a las 32 semanas de 34 días y a las 33 semanas tan sólo de 11 días. Esto nos indica que la duración promedio de la hospitalización es menor para los $\mathrm{RN}$ mayores a 32 semanas, lo que explica que algunos centros neonatales sólo reciban $\mathrm{RN}$ mayores a esta edad gestacional.

Las malformaciones congénitas (MC) por otra parte, constituyen un importante problema de Salud Pública, ya que en conjunto representan aproximadamente un $40 \%$ de la mortalidad perinatal. Se clasifican en mayores y menores, constituyendo un $2 \%$ y $14 \%$ respectivamente de los recién nacidos vivos del país. Sin embargo, esta incidencia se encuentra influenciada por el universo estudiado, representando $0,5-1$ de cada 10 mortinatos y 2 de cada 10 muertes infantiles.

A su vez, si consideramos las MC por sistemas, observamos que las más frecuentes son las cardiovasculares con un $18,9 \%$, seguida por las del sistema nervioso central (SNC) con un 16,5\% y posteriormente las gastrointestinales y genitourinarias con $9,1 \%$ y $5,4 \%$, respectivamente.

Por otra parte, si evaluamos la letalidad asociada a estas MC, observamos que las MC genitourinarias son las que presentan una mayor letalidad con un $65 \%$, seguidas por las MC del SNS con un $39,8 \%$ y posteriormente las MC gastrointestinales y cardiovasculares con un $21 \%$ y $12,3 \%$, respectivamente.

En la actualidad la pesquisa prenatal de MC se realiza a través de ultrasonido obstétrico, con una cobertura nacional de $60 \%$, lo que ha permitido aumentar la capacidad de detección de éstas, alcanzándose una sensibilidad cercana al $50 \%$ en el diagnóstico prenatal de MC. Esto ha generado un aumento en la derivación de embarazadas con fetos portadores de MC así como también de RN portadores de MC, durante las primeras 24 horas de vida, a centros de atención terciaria con Unidades de Cuidados Intensivos Neonatales, aumentando tanto el número de casos, como los gastos derivados de la atención de estos pacientes en estos centros.

Tanto los recién nacidos prematuros como los recién nacidos portadores de $\mathrm{MC}$ requieren de un mayor número de horas médicas, mayor número de días en UTI, medicamentos, insumos y procedimientos invasivos, que trae como consecuencia un costo final de hospitalización mayor, representando esto, para las instituciones proveedoras de salud, un impacto significativo desde el punto de vista económico.

\section{BIBLIOGRAFÍA}

1. $A B C$ of preterm birth: Epidemiology of preterm birth. Disponible en: http://www.studentbmj.com/issues/05/ 04/education/146.php. Consultado el 15 de diciembre de 2005.

2. Morgues $M$, Henríquez MT, Tohá $D$, Vernal $P$, Pittaluga E, Vega S, et al. Sobrevida del niño menor de $1500 \mathrm{~g}$ en Chile. Rev Chil Obstet Ginecol 2002;67(2): 100-5.

3. Águila $A$, Muñoz $H$. Tendencias de la natalidad, mortalidad general, infantil y neonatal en Chile desde 1850 a la fecha. Rev Méd Chil 1997;125:1236-45.

4. Águila A. Recién nacido de muy bajo peso de nacimiento, situación actual. Rev ChIL OBSTEt GINECOL 2000; 65:138-45.

5. Hubner ME, Ramírez R. Sobrevida, viabilidad y pronóstico del prematuro extremo. Rev Méd Chile 2002;130(8):931-8.

6. GRUPO COLABORATIVO NEOCOSUR. Very-lowbirh-weight infant outcomes in 11 South American NICUs. J Perinatol 2002;22(1):2-7. 